\title{
Quantitative measurement of amoxicillin in Ibuprofen tablets using UPLC
}

\begin{abstract}
A novel quantitative analytical method for the determination of Penicillin contaminant, Amoxicillin in non-penicillin pharmaceutical drug product (Ibuprofen tablet $400 \mathrm{mg}$ ) has been developed and validated using Ultra performance liquid chromatography (UPLC). The extraction of amoxicillin from the drug tablets was carried out with bi-distilled water and the separation was achieved by making use of a BEH C18 column with particle size of $1.7 \mu \mathrm{m}$ $(100 \mathrm{~mm} \times 2.1 \mathrm{~mm})$. The isocratic run accomplished using phosphate buffer $(\mathrm{pH} 5.0)$ : methanol $(95: 5, \mathrm{v} / \mathrm{v})$ mixture as mobile phase run at a flow rate of $0.3 \mathrm{~mL} / \mathrm{min}$. The rapid, accurate and low cost UPLC method was proven to be suitable within the current good manufacturing practices (cGMP) of pharmaceutical ingredients. In addition, the validation of the developed method was conducted as per the ICH (International conference of harmonization) guidelines Q2 (R1). Further, the method was found to be linear in the range of $\left(0.024-0.096 \mu \mathrm{g} / \mathrm{mL}\right.$ for amoxicillin) with a correlation coefficient, $R^{2}$ of 0.999 and net in terms of specificity, linearity, precision, accuracy, detection limit (DL), and quantitation limit (QL) are appeared to be satisfactory. The precision was assessed in terms of injections repeatability with a maximum $\%$ RSD of $1.8 \%$, while the intermediate precision Day-1 with $\%$ RSD of 0.96 and the intermediate precision Day-2 with \%RSD of 1.56 were observed. Thus, from the observation of satisfactory results for amoxicillin detection, the developed UPLC-based method can successfully be applied in the pharmaceutical quality control laboratories to fulfill the regulatory requirements.
\end{abstract}

Keyword: UPLC; Amoxicillin; Ibuprofen tablets; Quantitative determination; Penicillin traces; Pharmaceutical analysis 